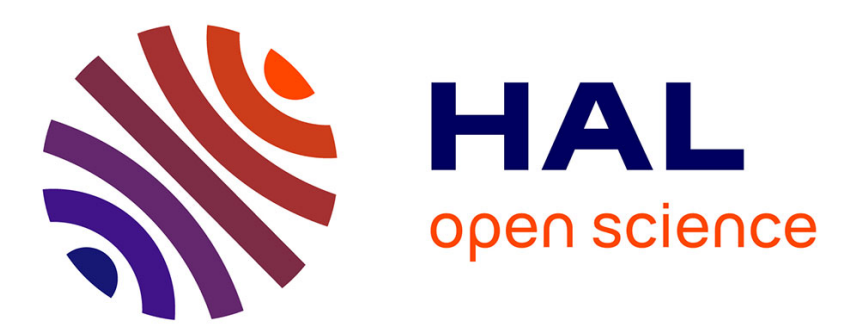

\title{
A particle-in-cell formulation for large deformation in Cosserat continua
}

\author{
Frédéric Dufour, Hans Muhlhaus, Louis Moresi
}

\section{To cite this version:}

Frédéric Dufour, Hans Muhlhaus, Louis Moresi. A particle-in-cell formulation for large deformation in Cosserat continua. Bifurcation and Localisation Theory in Geomechanics, 2001, Perth, Australia. hal-01007790

\section{HAL Id: hal-01007790 \\ https://hal.science/hal-01007790}

Submitted on 13 Jun 2019

HAL is a multi-disciplinary open access archive for the deposit and dissemination of scientific research documents, whether they are published or not. The documents may come from teaching and research institutions in France or abroad, or from public or private research centers.
L'archive ouverte pluridisciplinaire HAL, est destinée au dépôt et à la diffusion de documents scientifiques de niveau recherche, publiés ou non, émanant des établissements d'enseignement et de recherche français ou étrangers, des laboratoires publics ou privés. 


\title{
A particle-in-cell formulation for large deformation in Cosserat continua
}

\author{
F. Dufour, H.-B. Mühlhaus, L. Moresi \\ CSIRO Exploration and Mining, Perth, 6009, AUSTRALIA
}

\begin{abstract}
We present a new approach to modeling of granular flows using a combination of Cosserat theory for granular material and a particle-in-cell finite element method capable of handle extremely large material deformation. Benchmarking against analytical solutions highlights the strengths and weaknesses of the method. We demonstrate one application of the method in modeling the discharge of granular material from a silo.
\end{abstract}

\section{INTRODUCTION}

The mechanics of granular materials has intrigued physicists and engineers for well over two centuries. At low strain rates, particulates such as sand or cereals behave like solids, but at high strain rates, the behaviour is fluid or gas like.

A granular flow can be modeled by the Discrete Element Method (DEM) with a microscopic description of the particulate's behaviour (e.g. Sakaguchi et al. 1997). Unfortunately this method is not adapted to problems on the large scale or over long times. Numerous problems like this occur in the mining field where it is necessary to model the long-term behaviour (several years) of mine slopes which can be tens of meters high.

It is also possible to use a continuous approach with a parameter describing the microstructure of the material (internal length). The Cosserat theory (Cosserat 1909) is one of these. By adding rotational kinematic variables independant of linear variables we can model internal rotation of the material as well as couple stresses. This micropolar method can be used for every application which involves the need to describe the heterogenous microstructure of the material such as granular materials (e.g. Mühlhaus et al. 1991), layered materials (e.g. Adhikary et al. 1999) or crystals (e.g. Forest et al. 1997).

This class of methods can be implemented in the context of a classical finite element method (FEM). However, very large deformations are difficult to handle elegantly within the FEM because mesh distortion and remeshing can quickly present severe difficulties. The Particle-In-Cell (PIC) scheme is a hybrid numerical method which falls somewhere between the Finite Element Method (FEM) and a purely Lagrangian particle method such as DEM. The PIC scheme attempts to combine the versatility of the continuum FEM with the geometrical flexibility of DEM. A summary of this method as applied to fluid deformation with internal boundaries is given elsewhere in this volume by Moresi et al (2000).

In PIC we use Lagranian particles and an Eulerian mesh. The mesh is used to solve nodal point unknowns using almost exactly the same formulation as the standard FEM, however, the mesh is not required to track material deformation which avoids the problem of distortion. Our formulation is derived from fluid flow applications in which fluid velocities are solved on the mesh, and material strains are recorded by the Lagrangian particles. For this reason, our initial Cosserat implementation is a viscous formulation which should be seen as the first step towards a largedeformation viscoplastic formulation.

As with any new application of a numerical method, extensive benchmarking is needed to understand the characteristics of the algorithm including, for example, stability, convergence rates, and accuracy for a range of boundary conditions, material parameters, and internal interface geometries. We use a number of simple, analytic solutions to characterize the behaviour of the numerical scheme.

\section{MATHEMATICAL FORMULATION}

\subsection{Cosserat deformation measures}

We assume that deformation quantities are infinitesimal and assign a local rigid cross to every material point $\left(x_{1}, x_{2}, x_{3}\right)$ of the body in a Cartesian coordinate system $\left(X_{1}, X_{2}, X_{3}\right)$. In the process of deformation, the material points (rigid crosses) rotate at a rate $\omega_{i}^{c}$ about the axis $i$ in addition to the conventional linear velocity vector $\mathbf{u}$. The angular velocity $\omega_{i}^{c}$ is considered to be independent of $\mathbf{u}$ and differs from the 
classical rigid body angular velocity $\omega_{i}$,

$$
\omega_{i}=-\epsilon_{i j k} W_{j k}
$$

where

$$
W_{j k}=\frac{1}{2}\left(u_{j, k}-u_{k, j}\right)
$$

In (1) $\epsilon_{i j k}$ designates the permutation symbol, and in (2) $(.)_{, k} \equiv \partial(.) / \partial x_{k}$ are partial derivatives.

In the classical theory the stretching tensor is given by :

$$
D_{i j}=\frac{1}{2}\left(u_{i, j}-u_{j, i}\right)
$$

In the Cosserat theory, as a rotational parameter has been added, in addition to the classical strain rate tensor $\boldsymbol{D}$, there is an additional rate measure,

$$
\omega_{i}^{r e l}=\omega_{i}-\omega_{i}^{c}
$$

which represents the relative angular velocity between the material element and the associated rigid coordinate cross. In this case, the rate of the deformation tensor can be expressed by the rate of the distortion tensor, $\gamma$,

$$
\gamma_{i j}=u_{i, j}-W_{i j}^{c}
$$

where

$$
W_{i j}^{c}=-\epsilon_{k i j} \omega_{k}^{c}
$$

and by the tensor representing the measure of relative angular velocity between the neighbouring rigid crosses,

$$
\kappa_{i j}=\omega_{i, j}^{c}
$$

The conventional strain rate tensor can be expressed as the symmetrical part of the rate of the distortion tensor,

$$
D_{i j}=\frac{1}{2}\left(\gamma_{i j}+\gamma_{j i}\right)
$$

and the relative angular velocity as the antisymmetrical part

$$
W_{i j}^{r e l}=\frac{1}{2}\left(\gamma_{i j}-\gamma_{j i}\right)
$$

We have 2 deformation rate measures i.e. $\gamma$ and $\kappa$. Both measures are objective. In a rotating observer frame $\gamma$ and $\kappa$ are obtained as $Q \gamma Q^{T}$ and $Q \kappa Q^{T}$ where $Q Q^{T}=1$ describes the rotation of the moving -with respect to the fixed-observer frame.

\subsection{Constitutive relationships for granular materi- als}

As we want to model large deformation problems for visco-plastic Cosserat media, we have first implemented the Cosserat theory for a viscous fluid. In a 2D conventional continuum an isotropic material is characterised by a bulk viscosity $B$ and a shear viscosity $\eta$, for a Cosserat continuum we also have a Cosserat shear viscosity $\eta^{c}$ and a bending viscosity $M$. In other words, the relation between normal stresses and normal gradient of deformations remains the same as for conventional viscous medium whereas the relation between shear components is modified by $\eta^{c}$ and, in addition, the couple stress is related to Cosserat rotational velocity through the bending stiffness. The constitutive relation for a generalised Newtonian fluid can be written in the usual pseudo-vector form:

$$
\sigma=\Lambda D
$$

where the stress vector components are:

$$
\boldsymbol{\sigma}^{T}=\left\{\sigma_{x x}, \sigma_{y y}, \sigma_{x y}, \sigma_{y x}, \mu_{z x}, \mu_{z y}\right\}
$$

the deformation vector components are:

$$
D^{T}=\left\{\gamma_{x x}, \gamma_{y y}, \gamma_{x y}, \gamma_{y x}, \kappa_{z x}, \kappa_{z y}\right\}
$$

and the matrix $\Lambda$ is expressed as:

$$
\left\{\begin{array}{cccccc}
B+\eta & B-\eta & 0 & 0 & 0 & 0 \\
& B+\eta & 0 & 0 & 0 & 0 \\
& & \eta+\eta^{c} & \eta-\eta^{c} & 0 & 0 \\
& & & \eta+\eta^{c} & 0 & 0 \\
& \text { symm. } & & & M & 0 \\
& & & & & M
\end{array}\right\}
$$

The aim of this paper is the veryfication and demonstration of the Cosserat continuum implementation in the finite element code ELLIPSIS. For this purpose we use the simplest possible realisation of a granular, viscous medium. In the granular-elasticity model of (Choi and Mühlhaus 1991) we replace the contact stiffnesses $K_{n}$ and $K_{m}$ and relative displacements by contact dashpots $\eta_{n}$ and $\eta_{m}$ and relative velocities and relative rotation rates and obtain the relationships

$$
\begin{aligned}
\eta & =\frac{1-n}{4 \pi} k\left(\eta_{n}+\eta_{m}\right) \\
\eta^{c} & =\frac{1-n}{2 \pi} k \eta_{m} \\
B & =\frac{1-n}{2 \pi} k \eta_{n} \\
M & =2 \eta^{c} R^{2}
\end{aligned}
$$




\section{ANALYTICAL SOLUTIONS}

\subsection{Infinite shear Layer}

We extend the analytic solution of Choi \& Mühlhaus for flow in an infinite plane layer of Cosserat material subject to shearing top and bottom. To test the numerical stability of the code, we test all combinations of boundary conditions: $(\Omega, V),(\Omega, \sigma),(\mu, V)$ and $(\mu, \sigma)$.

The numerical solution is found using a rectangular 2D mesh (Fig. 1) with periodic boundary conditions an additional test of the accuracy of the code is the degree to which it reproduces a 1D solution throughout the mesh.

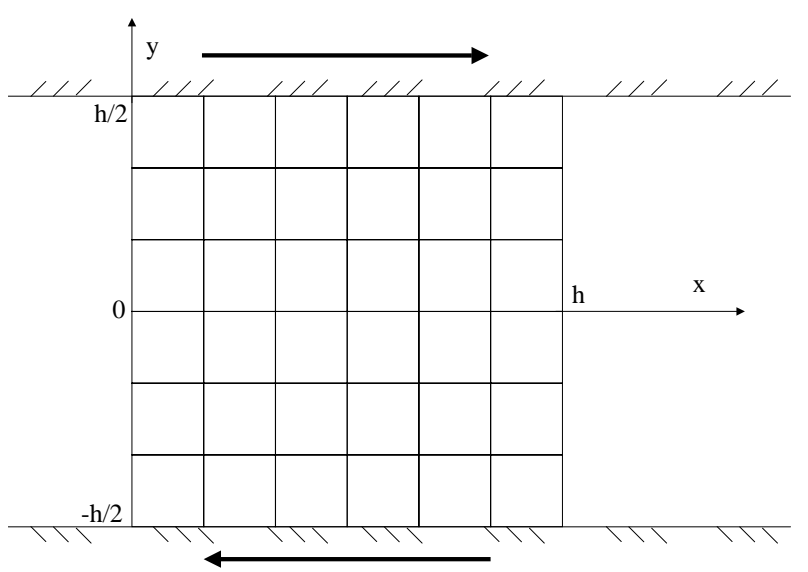

Figure 1: Geometry of the infinite shear layer

The governing equations are reduced as follows considering a 2D model as well as the independence of all variables on the $x$-direction.

$$
\sigma_{x y, y}=0
$$

and

$$
\mu_{z y, y}+\sigma_{x y}-\sigma_{y x}=0
$$

By appling the constitutive relationships between stresses and velocity gradients we get:

$$
\left(\eta+\eta^{c}\right) \frac{\partial^{2} u_{x}}{\partial y^{2}}-2 \eta^{c} \frac{\partial \omega_{z}^{c}}{\partial y}=0
$$

and

$$
M \frac{\partial^{2} \omega_{z}}{\partial y^{2}}-2 \eta^{c} \frac{\partial u_{x}}{\partial y}-4 \eta^{c} \omega_{z}^{c}=0
$$

Equations (20) and (21) are only coupled through $\eta^{c}$; when $\eta^{c}$ is zero, then the linear velocity reverts to the classical one. By integrating (20) and (21) with respect to $y$ we obtain the analytical solution for the linear velocity:

$$
\begin{aligned}
u_{x} & =-\frac{2 \eta^{c} \eta}{\eta+\eta^{c}}\left(b \sinh \frac{y}{\delta}+c \cosh \frac{y}{\delta}\right) \\
+\frac{a}{\eta} y+d &
\end{aligned}
$$

and the angular velocity:

$$
\omega_{z}=-\frac{a}{2 \eta}+b \sinh \frac{y}{\delta}+c \cosh \frac{y}{\delta}
$$

where a,b,c and d are integration constant and

$$
\delta^{2}=\frac{M}{4}\left(\frac{1}{\eta^{C}}+\frac{1}{\eta}\right)=\frac{R^{2}}{2}\left(1+\frac{\eta^{c}}{\eta}\right)
$$

Due to the symmetry of the model, $c=d=0-$ other constants are computed according to the boundary conditions listed in Figure 2.

\subsection{Gravity driven flow}

A second analytic solution is required to ensure that flow driven by body forces can be computed accurately. We specify zero rotation and velocity on top and bottom, which models a non-frictional boundary $(v=0)$ and a rough surface $(w=0)$ (Tejchman 1992). As a driving force we apply a body force, $g$, positive in the $x$-direction. In this particular model the linear velocity is symmetric about the mid-line whereas the Cosserat rotation is antisymmetric then (18) becomes:

$$
\sigma_{x y, y}=\rho g
$$

The same procedure as previously described yields to the analytical expression for linear velocity:

$$
u=\rho g \frac{y^{2}}{2 \eta}-\frac{2 \eta^{c}}{\eta+\eta^{c}} b \delta \cosh \frac{y}{\delta}+c
$$

where a and c are integration constants and for rotational velocity:

$$
\omega=-\frac{\rho g y}{2 \eta}+B \sinh \frac{y}{\delta}
$$

with

$$
B=\frac{\rho g h}{4 \eta \sinh \frac{h}{2 \delta}}
$$

and

$$
C=\frac{2 \eta^{c}}{\eta+\eta^{c}} B \delta \cosh \frac{h}{2 \delta}-\rho g \frac{h^{2}}{8 \eta}
$$




\begin{tabular}{|c|c|c|}
\hline B.C. type & Value of $a$ & Value of $b$ \\
\hline $\begin{array}{c}u_{x}\left(\frac{h}{2}\right)=-u_{x}\left(-\frac{h}{2}\right)=V \\
\omega_{z}\left(\frac{h}{2}\right)=\omega_{z}\left(-\frac{h}{2}\right)=\Omega\end{array}$ & $2 \eta\left(b \cosh \frac{h}{2 \delta}-\Omega\right)$ & $\frac{V+\Omega h}{h \cosh \frac{h}{2 \delta}-2 \delta \frac{\eta^{c}}{\eta+\eta^{c}} \sinh \frac{h}{2 \delta}}$ \\
\hline $\begin{array}{c}u_{x}\left(\frac{h}{2}\right)=-u_{x}\left(-\frac{h}{2}\right)=V \\
\mu_{z y}\left(\frac{h}{2}\right)=\mu_{z y}\left(-\frac{h}{2}\right)=\mu\end{array}$ & $\frac{2 \eta}{h}\left(V+2 b \delta \frac{\eta^{c}}{\eta+\eta^{c}} \sinh \frac{h}{2 \delta}\right)$ & $\frac{\mu \delta}{M \sinh \frac{h}{2 \delta}}$ \\
\hline $\begin{array}{c}\sigma_{x y}\left(\frac{h}{2}\right)=-\sigma_{x y}\left(-\frac{h}{2}\right)=\sigma \\
\omega_{z}\left(\frac{h}{2}\right)=\omega_{z}\left(-\frac{h}{2}\right)=\Omega\end{array}$ & $\sigma$ & $\frac{\sigma}{2 \eta \cosh \frac{h}{2 \delta}}$ \\
\hline $\begin{array}{c}\sigma_{x y}\left(\frac{h}{2}\right)=-\sigma_{x y}\left(-\frac{h}{2}\right)=\sigma \\
\mu_{z y}\left(\frac{h}{2}\right)=\mu_{z y}\left(-\frac{h}{2}\right)=\mu\end{array}$ & $\sigma$ & $\frac{\mu \delta}{M \sinh \frac{h}{2 \delta}}$ \\
\hline
\end{tabular}

Figure 2: Table of parameters.

\section{BENCHMARKING}

The equation solver in our code is a multigrid-based iterative scheme. This requires the specification of an error tolerance which we choose to be smaller than the expected discretization error - in this case a value of $10^{-6}$ was felt to be sufficient. If not specified the integration over an element is a $2 \times 2$ scheme of evenly distributed and weighted particles. In post-processing, error is computed on a nodal field $\phi$ as follows:

$$
\operatorname{Err}(\phi)=\frac{\sum_{i=1}^{n n o}\left|\phi_{i}^{\text {anal }}-\phi_{i}^{\text {num }}\right|}{\sum_{i=1}^{n n o}\left|\phi_{i}^{\text {num }}\right|}
$$

where nno is the number of nodal points in the mesh. Numerical values for material parameters used in (14) are:

$$
\begin{array}{rlrl}
K_{n} & =K_{m}=10^{5} \mathrm{MPa} & k=4, \\
R & =0.3 \mathrm{~m} & n=0
\end{array}
$$

with this set of parameters $\eta=\eta^{c}$ which means that the rotational part is fully coupled with the linear part and the internal length is about $1 / 3$ the layer thickness. All the following results are valid from the first timestep (Fig. 3.a) to the one thousand timestep (Fig. 3.d) which is indicative of the insensitivity of the scheme to large deformations. In Figure 3.a and $3 . b$ only one black band is in the sample cell of our periodic model, while the deformation keep growing the neighbour bands come into the cell from edges. We then always have an odd number of bands and the initial band remains in the middle of the cell.

\subsection{F.E.M. convergence.}

In order to demonstrate that in ELLIPSIS the numerical solution converges to the true solution as the number of elements becomes infinite, we plot against the

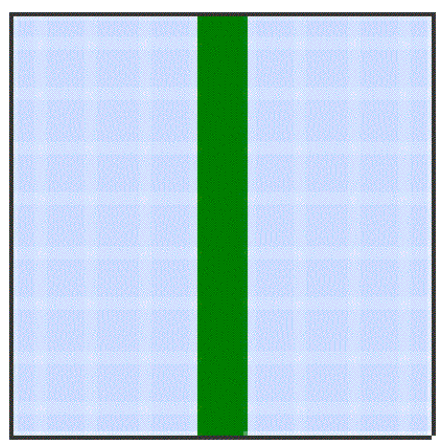

(a)

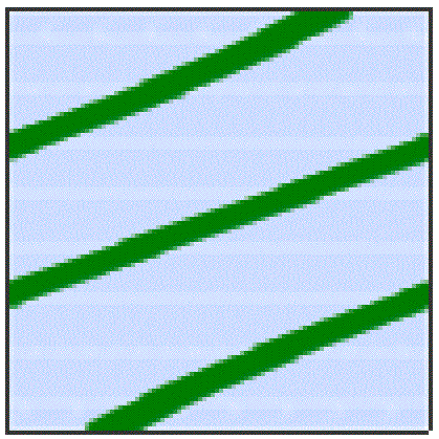

(c)

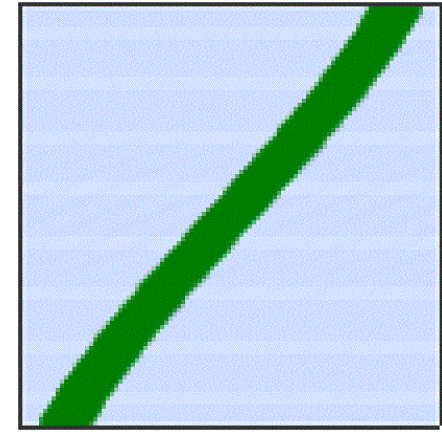

(b)

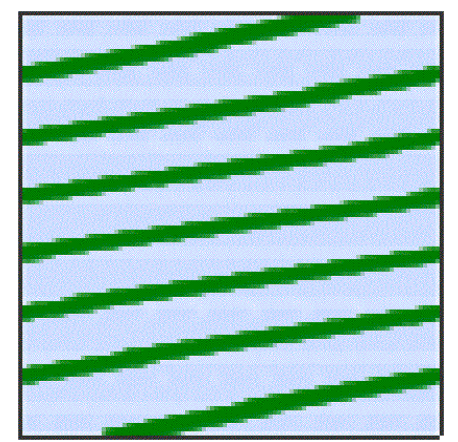

(d)
Figure 3: Snapshots of the model. (a) $t=0$ sec., (b) $t=7.110^{4} \mathrm{sec} .,(\mathrm{c}) t=1.210^{6} \mathrm{sec}$. and (d) $t=$ $5.7410^{6}$ sec..

number of elements the ratio defined by the error on the current grid over the error on the coarsest grid. For that we use 5 different meshes in which the number of elements in the $x$-direction is successively 6 , 12, 24, 48 and 96. The error is scaled to avoid any dependency on other variables such as magnitude of boundary conditions.

All the curves lie very close together and the convegence rate is approximately the same magnitude for linear velocity (Fig. 4) and angular velocity (Fig. 5). For other computations we will use the 24 element mesh. 


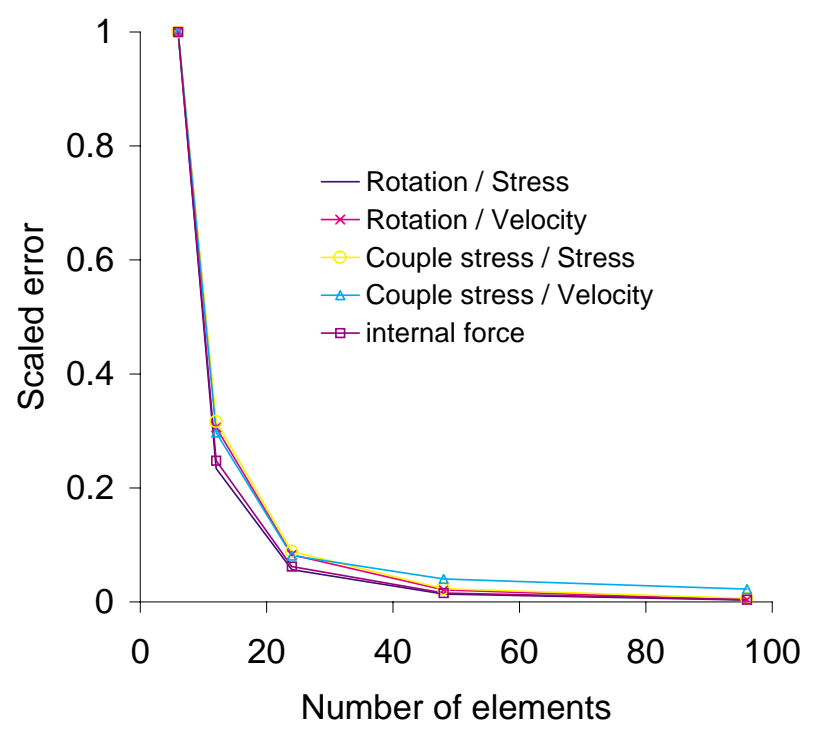

Figure 4: Ratio of the error on linear velocity for different driving boundary conditions.

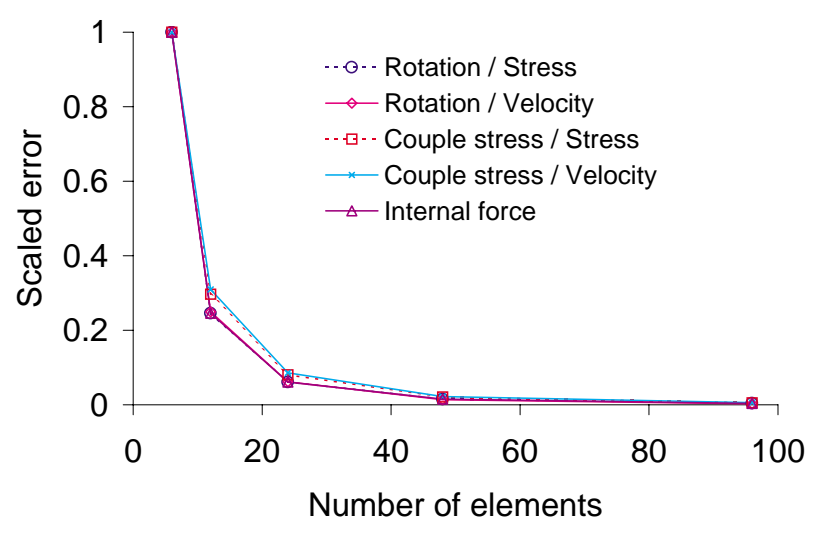

Figure 5: ratio of the error on rotation for different driving forces.

\subsection{Significance of non-standard boundary condi- tions.}

An unusual situation arises in this problem: Depending on the relative magnitude of the boundary conditions on velocity compared to rotation, we can model a true Cosserat medium or we can be in a domain where we deal essentially with a classical continuum. For instance, in the case where we prescribe linear stress and angular velocity as boundary conditions, if the magnitude of the rotational boundary condition is exactly the same as the one resulting from the classical continuum theory then the solution is the classical one. We present only the case of $(\omega, \sigma)$ (Fig. 6) and $(\mu, v)$ (Fig. 7) since the cases $(\mu, \sigma)$ and $(\Omega, V)$ are similar to $(\mu, V)$ and $(\Omega, \sigma)$ respectively. In Figure 6 and $7, k$ and $j$ represent dimensionless parameters defined as:

$$
j=\frac{\mu}{v M R^{2}} \quad \text { and } \quad k=-\frac{\sigma}{2 \eta \omega}
$$

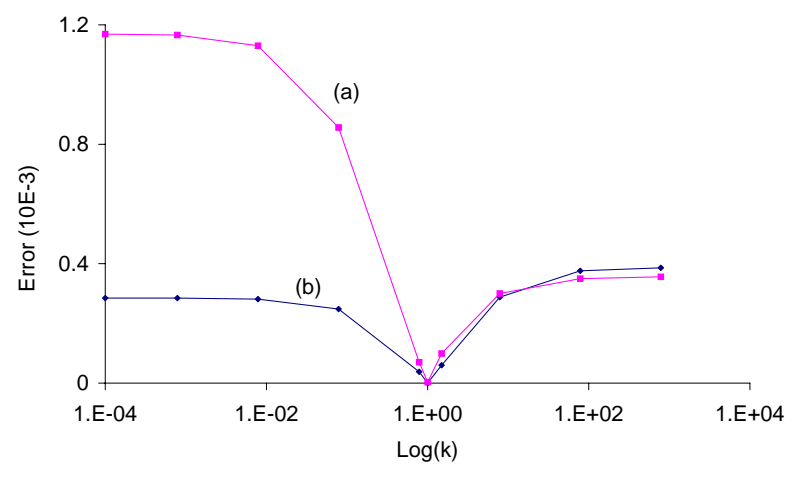

Figure 6: Error on linear velocity (a) and angular velocity (b), for $(\Omega, \sigma)$ boundary condition case.

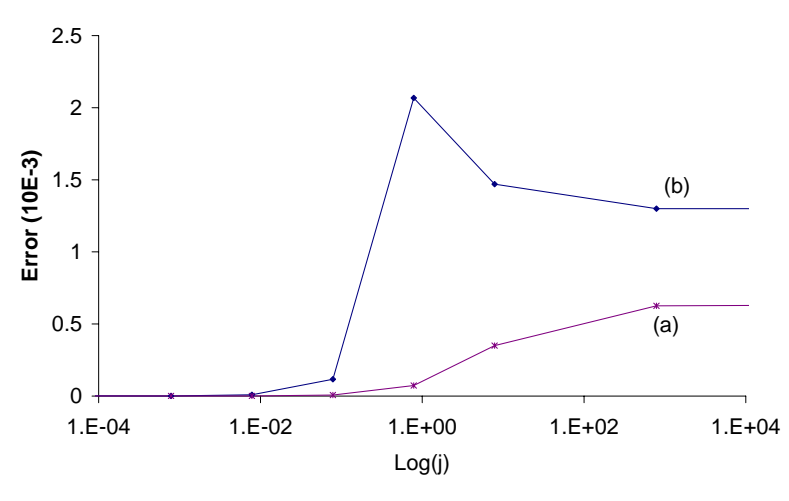

Figure 7: Error on linear velocity (a) and angular velocity $(\mathrm{b})$, for $(\mu, \mathrm{V})$ boundary condition case.

Figure 6 presents two distinct parts, one point where the solution is 'exact' (error has same magnitude than numerical accuracy), and two half-spaces where error are quite small but not in the same magnitude. At this particular point we have $\sigma_{x y}=-2 \eta \omega_{z}$ which corresponds to the constant rotation of the classical continuum. Bilinear elements are capable of reproducing the exact solution in this case. On the other hand when $\omega^{c} \neq \omega$ then the solution becomes less accurate. We are confident that our implementation is accurate for the Cosserat continuum since the results of Figure 6 show that the error for either velocity or rotation never exceeds $0.12 \%$. Figure 7 looks different but we still can distinguish two distinct parts. One half-space where we get the exact solution and one half-space where the error on rotation can reach $0.2 \%$. The latter corresponds to a true Cosserat continuum whereas in the first part the couple stress is tiny and then we can consider in that domain the continuum as a non-polar 
one.

\subsection{Internal length effects}

For the case where $\Omega$ and $\sigma$ are applied as boundary conditions and the material is fully coupled $\left(\eta=\eta^{c}\right)$, we plot (Fig. 8) the velocity on the top (maximum value) against the internal length. In an elastic contin-

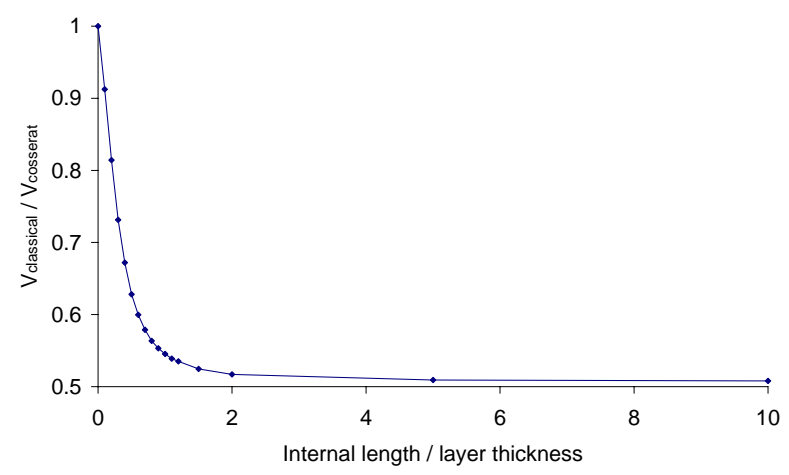

Figure 8: Variation of the flow velocity Vs. the grain size.

uum the displacement is maximum for $R=0$ (classical continuum) and decreases to zero as $R$ reaches the characteristic size of the mesh (layer thickness). In a viscous Cosserat medium the flow velocity is maximum for $R=0$ and tends to half the maximum value when $R \rightarrow \infty$. The plot shows a clear difference in the result depending whether $R \geq h$ or $R \leq h$. Rewriting (22) and taking into account the appropriate integration constants gives

$$
u_{x}=\frac{\sigma h}{2 \eta}\left(1-\frac{R}{h} \tanh \left(\frac{h}{2 R}\right)\right)
$$

We can easily demonstrate through the equivalence of tanh in infinity that the outflow for a classical continuum is twice faster than a outflow of a infinite internal length Cosserat continuum in the case of a fully coupled material. We can explain this phenomenon by the co-existence of two differrent modes of flow. The first one is due to a relative displacement between grains through a frictional contact. The second one is only due to the grain viscosity itself. Then for $R=0$ the flow is the fastest because it is governed by vicosity of the grain as well as a relative rotation between grains. The last effect becomes less effective as the internal length increases, then the flow slows down. Finally when $R \geq h$, the flow is minimum and does not change anymore with the variation of the internal length. The rearrangement effect between grains is no longer possible because of the bending stiffness and only the viscous flow is present.
Worth noting is the fact that with no elastic term we can not expect the formation of an arch to stops the flow for an internal length larger than the characteristic size of the system.

\section{APPLICATION}

We now consider the discharge of a Cosserat material from a model silo. In this case we are interested in the influence of the internal length parameter on the velocity flow for a Cosserat fluid. Figure 9

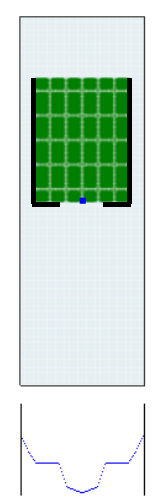

(a)

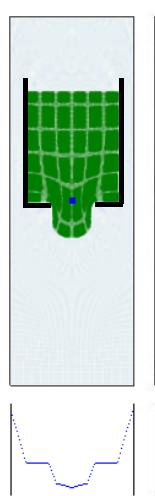

(b)

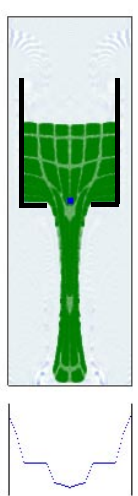

(c)

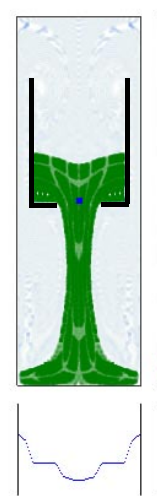

(d)

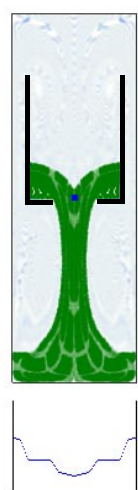

(e)
Figure 9: Snapshots of the model. (a) Initial conditions, (b) $t=4.7510^{-4}$ sec., (c) $t=1.4210^{-3}$ sec., (d) $t=2.8510^{-3}$ sec. and (e) $t=3.810^{-3}$ sec.

shows the geometry of the model as well as different snapshots along the computations. All the heavy lines are free-slip boundaries and the flow is only due to the downward gravity field. The grid drawn on the flowing material is a "dye" to record deformation it does not affect the material properties. Corridors along the edges provide space where upward flow of the background material (similar to air) can take place to equilibrate the pressure due to the downward flow of the Cosserat viscous material. The mechanical characteritics of each material are summarized in Figure 10. In Figure 11 we plot the volume flowing

\begin{tabular}{|c|c|c|}
\hline & Granular material & Background \\
\hline Internal length & $R$ & 0 \\
\hline Shear viscosity & 1000 & 1 \\
\hline Bulk viscosity & $+\infty$ & $+\infty$ \\
\hline Density & $10^{6}$ & 0 \\
\hline
\end{tabular}

Figure 10: Constant values.

out the reservoir versus time and for different values of the ratio $\alpha=R / a$. The flow rate is almost identical for all values of $\alpha$ larger than the aperture (1.0) which corresponds, in the elastic case, to a situation in which no flow can occur. Note that in purely viscous materials static equilibrium states do not exist. Consequently the flow arrest through arching for instance 
can not be expected. For $0 \leq \alpha \leq 1.0$, the smaller the internal length the faster the outflow. As for the elastic case the internal length provides a bending stiffness which slows down the flow.

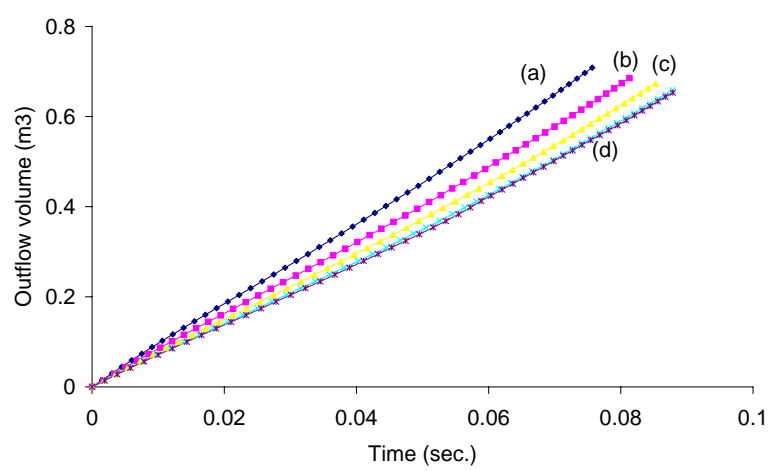

Figure 11: Outgoing volume for (a) $\alpha=0$, (b) $\alpha=1 / 3$, (c) $\alpha=2 / 3$, (d) $\alpha=5 / 3$ and (e) $\alpha=10 / 3$

\section{CONCLUDING REMARKS}

We have implemented a Cosserat viscous formulation into a PIC code which allows us to reproduce qualitatively a 1-D granular flow in large deformation. Results on the silo discharged problem are well in accordance to experimental tests.

\section{REFERENCES}

D.P. Adhikary, H.B. Mühlhaus and A.V. Dyskin (1999), Modelling the large deformations in stratified media - the Cosserat continuum approach. $M e$ chanics of Cohesive-Frictionnal Materials, 4, 195213.

S.K. Choi and H.B. Mühlhaus (1991), Distinct element vs Cosserat theory: A comparison for the case of an infinite shear layer. Computer Methods and Advanced in Geomechanics, Beer, Booker \& Carter (eds), Balkema, 315-319.

E. Cosserat and F. Cosserat (1909), Théorie des corps déformables.Hermann (ed.), Paris.

S. Forrest, G. Cailletaud and R.Sievert (1997), A Cosserat theory for elastoviscoplastic single crystals at finite deformation. Arch. Mech., 49, 4, pp 705-736.

L.N. Moresi and V.S. Solomatov (1995), Numerical investigation of 2D convection with extremely large viscosity variations. Phys. fluids, Vol 7, No 9, pp 2154-2162.
L.N. Moresi, H.-B. Mühlhaus and F. Dufour (2000), Particle-in-cell solutions for creeping viscous flows with internal interfaces. *************************************

H.B. Mühlhaus, R. de Borst and E.C. Aifantis (1991), Constitutive models and numerical analyses for inelastic materials with microstructure. Computer Methods and Advanced in Geomechanics, Beer, Booker \& Carter (eds), Balkema, 377-385.

H. Sakaguchi, A. Murakami and T. Hasegawa (1997), Vortex pattern and its diameter in relation to the thickness of shear bands in granular materials. Deformation and progressive failure in geomechanics, Asaoka, Adachi \& Oka (eds), Pergamon, 93-96.

J. Tejchman (1992), Behaviour of a granular medium in a silo - model tests in a plane silo with parallel walls, part I. Archives of Civil Engineering, XXXVIII, 4. 